ISSN: 2386-3919 - e-ISSN: 2386-3927

DOI: https://doi.org/10.14201/et201735199116

\title{
DESARROLLO DEL PLURILINGÜISMO A TRAVÉS DEL PORTFOLIO EUROPEO DE LAS LENGUAS ELECTRÓNICO (E-PEL) EN LA ESCUELA OFICIAL DE IDIOMAS: ESTUDIO DE UN CASO
}

\author{
Development of plurilingualism through the Electronic \\ European Language Portfolio (eELP) at the Official \\ Language School: a case study
}

\author{
Mario Jesús MiRA-GimÉNEZ \\ EOI Alicante.Correo-e: mira_margim@gva.es
}

Recibido: 08/02/2016; Aceptado: 15/03/2017; Publicado: 31/05/2017

Ref. Bibl. MARIO JESÚS MIRA-GIMÉNEZ. Desarrollo del plurilingüismo a través del Portfolio Europeo de las Lenguas Electrónico (e-PEL) en la Escuela Oficial de Idiomas: estudio de un caso. Enseñanza \& Teaching, 35, 1-2017, 99-116.

RESUMEN: Las TIC han revolucionado nuestra forma de interactuar e informarnos, de tal manera que la comunicación plurilingüe e intercultural se ha convertido en imprescindible en la Sociedad de la Información, aspecto que propugna el MCERL (Marco Común Europeo de Referencia para las Lenguas). A tal efecto, se han puesto en práctica algunas iniciativas europeas al respecto con diferentes grados de repercusión. Este artículo presenta los resultados de una investigación sobre el e-PEL (Portfolio Europeo de las Lenguas Electrónico). El objetivo ha consistido en analizar el desarrollo del plurilingüismo a través de este documento electrónico del Consejo de Europa, en lo que respecta a actitudes y actividades plurilingües. El estudio se ha llevado a cabo durante seis meses, de noviembre de 2015 a abril de 2016, en cuatro grupos de alemán de nivel A2.1, B1.1 y B1.2 de la Escuela Oficial de Idiomas de Alicante, con un total de 109 alumnos, si bien los resultados se han analizado de forma global, independientemente del grupo. Se ha utilizado una metodología cuantitativa con un pretest y un postest: los estudiantes han cumplimentado el cuestionario de 
los 18 descriptores de plurilingüismo del e-PEL antes y después de la aplicación para averiguar el grado de consecución de los objetivos. Los datos se han analizado de forma global y desagregados por sexo y grupos de edad. A tenor de los resultados, se puede concluir que el e-PEL ha contribuido al desarrollo del plurilingüismo, especialmente en las actividades plurilingües. No obstante, se necesitaría más tiempo para implementar las actitudes en favor de la variedad lingüistica y cultural, en especial entre los varones y los grupos de edad de menos de 20 años y de más de 50 años.

Palabras clave: Plurilingüismo; Interculturalidad; e-PEL; Aprendizaje de lenguas.

SUMMARY: ICT have revolutionized the way we interact and inform, so that the multilingual and intercultural communication has become essential in the Information Society, an aspect that advocates the CEFR (Common European Framework of Reference for Languages). To this end, some European initiatives have been implemented in this regard with varying degrees of impact. This article presents the results of a research on the eELP (Electronic European Language Portfolio). The aim has been to analyze the development of multilingualism through this electronic document of the Council of Europe, as to attitudes and multilingual activities. The study was conducted for six months, from November 2015 to April 2016 in four groups of German students (levels A2.1, B1.1 and B1.2) at the Official School of Languages in Alicante, with a total of 109 students. A quantitative methodology was used with a pretest and posttest: students filled out a questionnaire of the 18 descriptors on multilingualism on the eELP before and after the implementation to find out the degree of achievement of goals. The data were analyzed globally and broken down by sex and age groups. According to the results, it can be concluded that the eELP has contributed to the development of multilingualism, especially in multilingual activities. However, it would take more time to implement attitudes towards linguistic and cultural variety, particularly among men and the age groups of less than 20 years and more than 50 years.

Key words: Plurilingualism; Interculturality; eELP; Language learning.

\section{INTRODUCCIÓN}

El mapa lingüístico de todo el mundo indica una gran diversidad en cuanto a los idiomas y dialectos hablados y escritos en varios países y regiones, por lo que la comunicación plurilingüe e intercultural se ha convertido en un tándem conceptual que necesita interpretaciones coherentes y orientadas a la acción, lo que conduce a políticas y estrategias pertinentes y eficaces a nivel local, nacional, regional y global (Bârliba, 2015). Esta realidad dinámica no se ha reflejado en la enseñanza-aprendizaje tradicional, que presenta «una orientación general que refleja claramente un enfoque modernista, en el cual las culturas meta se basan en una visión homogénea, entre estable y estática y de carácter nacional sobre algunos de los países donde se utiliza la lengua meta» (López y Abello, 2016: 217). 

EN LA ESCUELA OFICIAL DE IDIOMAS: ESTUDIO DE UN CASO

En el período de recesión económica acompañada de una crisis de valores y el aumento de las tensiones interculturales, la enseñanza-aprendizaje de idiomas y la formación del profesorado deben convertirse en un sistema dinámico, que se debe enfrentar a muchos desafíos (Bien, Merten y Schnotz, 2015). El mundo globalizado actual requiere una mayor flexibilidad en los programas de formación del profesorado para la enseñanza de idiomas (Pérez, 2016) en todos los grupos de edad, tanto en la enseñanza reglada como en el aprendizaje a lo largo de la vida a través de las Tic, tanto en el ámbito personal como profesional (Komorowska, 2016), «ya que la capacidad de comunicación es el primer requisito que ha de cumplir el individuo para desenvolverse en un contexto recientemente pluricultural y plurilingüe» (Real Decreto 126/2014, Anexo 1).

\section{MARCO TEÓRICO}

Diferentes teorías han tenido en cuenta los aspectos interculturales en la enseñanza-aprendizaje de lenguas, tales como la política de reconocimiento (Taylor 1993), interdependencia lingüística (Cummins, 1983), competencia comunicativa intercultural (Byram, 1992) y los prosumidores interculturales (Dezuanni y Monroy-Hernández, 2012). De estos estudios se desprende que los adultos bilingües realizan asociaciones cuando aprenden una L3, especialmente en edades tempranas si se fomenta la reflexión interlingüística. Las ventajas no se limitan a las similitudes y diferencias entre idiomas, sino que se amplían al uso de estrategias y mayor conciencia metalingüística sobre el aprendizaje. El sujeto plurilingüe puede convertirse en el modelo de enseñanza, que implica que los profesores se centran en la capacidad lingüística general. De esta manera, se facilita la reflexión (Collins y Muñoz, 2016; Jessner, Allgäuer-Hackl y Hofer, 2016; Lindorfer, 2016).

El plurilingüismo conlleva de igual manera otras ventajas. En primer lugar, todas las lenguas, también las minoritarias, constituyen un patrimonio lingüístico y cultural. En segundo lugar, los individuos plurilingües gozan de ventajas sociales, lingüísticas y cognitivas, las cuales se pueden traducir en mayor rendimiento escolar. Por último, el plurilingüismo posibilita la participación de los individuos en la Sociedad de la Información (Block, 2016; Fortanet-Gómez, 2013; Hancock, 2014).

El MCERL pretende superar a través de sus principios las barreras del idioma en una multilingüe Europa a través de la promoción del aprendizaje de idiomas, en general, y del plurilingüismo, en particular (Nieuwboer y van't Rood, 2016). A tales efectos, el e-PEL podría fomentar esta diversificación en la oferta de lenguas de las instituciones educativas, de tal manera que los estudiantes tengan la oportunidad de desarrollar la competencia plurilingüe. El pluriculturalismo subyace en el plurilingüismo, ya que los idiomas son aspectos indispensables de las culturas y la competencia comunicativa requiere el conocimiento de la cultura (North, 2016). Este aspecto contenido en el currículum de la EOI necesita un documento interlingüístico como el e-PEL donde los alumnos registren su progreso, ya que la pluriculturalidad implica identificarse con los valores de varias culturas, así como la 

EN LA ESCUELA OFICIAL DE IDIOMAS: ESTUDIO DE UN CASO

adquisición de las competencias lingüísticas necesarias para participar activamente en los intercambios culturales (López-Rocha, 2016).

Asimismo, plurilingüismo y pluriculturalismo promueven el desarrollo de la conciencia lingüística y comunicativa (Angelova y Zhao, 2016). Por consiguiente, ambos tienen una relación de interdependencia. A tales efectos, el MCERL propugna el plurilingüismo como objetivo de aprendizaje y propone la realización de tareas y actividades encaminadas al desarrollo de la competencia plurilingüe y pluricultural durante la enseñanza-aprendizaje de un idioma extranjero, de acuerdo con las tesis de Zorba y Arıkan (2016). La EOI cumplió cien años en 2011. Esta institución pionera en la enseñanza especializada de lenguas, que se ha convertido en una red de 300 centros distribuidos por todo el país e imparte clases de 23 lenguas, es un contexto idóneo para ser un referente de plurilingüismo.

De hecho, uno de los pilares de la política lingüística del Consejo de Europa se fundamenta en el plurilingüismo, que se plasma en iniciativas como el MCERL y su aplicación práctica, el Portfolio Europeo de las Lenguas (PEL). La interculturalidad se convierte, por tanto, en método de aprendizaje (Álvarez, 2016). La versión electrónica del PEL, el e-PEL (Portfolio Europeo de las Lenguas Electrónico), consta también de tres secciones (González, 2015):

- Pasaporte de las Lenguas: Plantilla de autoevaluación de las competencias lingüísticas a través de certificados y experiencias lingüísticas e interculturales.

- Biografía lingüística: Registro de las experiencias del usuario, cuyo objetivo reside en la planificación y evaluación del proceso de aprendizaje.

- Dosier: Recopilación digital de experiencias de aprendizaje y capacidades del estudiante en diferentes formatos.

El e-PEL como herramienta, por tanto también colectiva, transciende un carácter meramente lingüístico, adquiriendo un enfoque transversal gracias a su función informativa (movilidad académica y profesional), didáctica (competencia plurilingüe e intercultural a través del aprendizaje cooperativo) y reflexiva (aprendizaje estratégico y significativo a lo largo de la vida). La innovación que supone el e-PEL no radica en sus principios y funciones individuales, sino en la forma en que estos se interrelacionan, implicando directamente al alumno tanto en el proceso como en el resultado. El e-PEL está destinado a aprendientes de idiomas adultos que, en Universidad o en la EOI, necesitan seguir aprendiendo lenguas y aprender a mantener estos conocimientos de forma autónoma y significativa a lo largo de la vida, para lo cual el e-PEL podría constituir una herramienta eficaz para el desarrollo de la competencia plurilingüe e intercultural, de acuerdo con las tesis de Schärer (2015).

Para valorar la aportación del e-PEL, es necesario tener en cuenta otras iniciativas europeas encaminadas al fomento de la interculturalidad. El Europass es un portfolio, de cinco documentos, cuyo objetivo radica en plasmar las aptitudes y las calificaciones del usuario, fomentando la movilidad laboral y académica y, por 

EN LA ESCUELA OFICIAL DE IDIOMAS: ESTUDIO DE UN CASO

consiguiente, el intercambio lingüístico y cultural. En la actualidad está disponible en 26 idiomas (Konstantinidou y Pampouri, 2015).

Una de las últimas propuestas del Consejo de Europa es Erasmus +. La acción clave 1 incluye las iniciativas referentes a la movilidad encaminada al aprendizaje, mientras que la acción clave 2 engloba las iniciativas de cooperación para la innovación y el intercambio de buenas prácticas. Ambas perspectivas confluyen en «nuove prospettive per l'inserimento professionale ed evidenziano da un lato esigenze di formazione a livello della comunicazione internazionale e interculturale e dall'altro la necessità di sviluppare di capacità a lavorare in team multilingue e multiculturali» (Garbarino, 2015: 85).

Otra iniciativa de la UE es el Sello Europeo para las iniciativas innovadoras en la enseñanza y aprendizaje de las lenguas es una distinción de la Unión Europea para experiencias innovadoras en la enseñanza-aprendizaje de las lenguas, al tiempo que sensibiliza sobre las lenguas minoritarias y regionales, lo que entronca con uno de los propósitos del e-PEL (Mira-Giménez, 2015).

El proyecto MAREP (Marco Común de Referencia para los Enfoques Plurales de las Lenguas y de las Culturas), del Centro Europeo de Lenguas Modernas (Consejo de Europa), ha desarrollado un instrumento para transmitir el enfoque plural en el aula mediante una lista de descriptores sobre plurilingüismo e interculturalidad y una serie de materiales y recursos para su implementación. Esta herramienta incluye todos los idiomas y variedades, ofreciendo por tanto recursos para transmitir los principios teóricos de las políticas lingüísticas en práctica (Daryai-Hansen, Gerber, Lörincz, Haller, Ivanova, Krumm y Reich, 2015).

Asimismo, la iniciativa 1+2 de la Unión Europea se ha traducido en programas AICLE (Aprendizaje Integrado de Contenido y Lengua), los cuales se circunscriben en la mayoría de casos a educación bilingüe español-inglés (Lancaster, 2016). Sin embargo, se debe considerar la importancia de la calidad sobre la cantidad de exposición a la lengua extranjera para un aprendizaje significativo (Meyer, Prediger, César y Norén, 2016).

En consonancia con estas propuestas, el e-PEL fomenta la integración de los intercambios lingüísticos y culturales en la enseñanza-aprendizaje de idiomas. Implementados a través de las Tic, tienen lugar con independencia espacial y temporal mediante la interacción audiovisual con miembros de otras culturas, lo cual redunda en un aumento de la motivación gracias a intercambio con interlocutores reales. Desde la perspectiva cognitiva, se promueve la concienciación de la interculturalidad, a través del aprendizaje en situaciones reales (Nicolás y Abril, 2015; Serrador, 2015). El enfoque plurilingüe del e-PEL supone una perspectiva holística de competencias múltiples, en la que subyace la interdependencia de los idiomas en la mente y la interacción entre el desarrollo lingüístico, cognitivo y cultural (Hanesová, 2015). La perspectiva interlingüística e intercultural converge, por tanto, con el medio digital en línea, contribuyendo a un triple desarrollo de competencias básicas: lingüística, cultural y digital (Anderson y Macleroy, 2016). 

EN LA ESCUELA OFICIAL DE IDIOMAS: ESTUDIO DE UN CASO

El aprendizaje mediante el e-PEL se basa en el trabajo cooperativo, que se traduce en una mejor gestión del aula en términos de motivación, implicación y diversidad (Kabilan, 2016) a través del plurilingüismo (Iñesta y Pascual, 2015). Su aplicación implica un modelo alternativo asumido por la comunidad educativa que se refleja en el Proyecto Educativo de Centro (PEC). La atención a la diversidad cultural y lingüística implica comprender la interculturalidad como realidad enriquecedora, que tiene su reflejo en el respeto del plurilingüismo en un currículo intercultural (García y Moreno, 2014; Mira-Giménez, 2016).

El e-PEL, asimismo, contribuye al cambio de perspectiva, centrada en el alumno. En el ámbito específico de la tarea docente, un ejemplo al respecto es el EPOSTL (European Portfolio for Student Teachers of Languages) (Newby, 2007; Newby, Fenner y Jones, 2011), que promueve una reflexión sistemática sobre el conocimiento y la didáctica, evaluando sus propias competencias pedagógicas mediante un registro de su experiencia docente (Díaz, 2016; Dumbavescu y Merino, 2015). El e-PEL tiene tres enfoques pedagógicos, basados en el desarrollo de la autonomía del alumno, la conciencia/competencia intercultural y el plurilingüismo; y dos funciones, pedagógica e informativa. Su vinculación con el MCERL (Marco Común Europeo de Referencia para las Lenguas) se concreta en el alumno como centro del proceso de aprendizaje, en la determinación de objetivos y en listas de autoevaluación de descriptores, según competencias y niveles (Little y Erickson, 2015). El e-PEL entronca, por consiguiente, con la Autobiografía de Encuentros Interculturales (AEI) del Consejo de Europa, encaminada al desarrollo de la competencia intercultural (Messina, 2015). Con la aplicación de las TIC, la AEI se ha convertido en la Autobiografía de Encuentros Interculturales a través de los Medios Visuales (AEIMV), que se centra en experiencias interculturales en la Sociedad de la Información (Méndez, 2016). En consonancia con el e-PEL, los usuarios desarrollan actitudes, destrezas y conocimientos interculturales, a través de la reflexión y la conciencia de uno mismo. Su enfoque considera el proceso, así como el producto, integra los procedimientos de aprendizaje y evaluación, fomenta la reflexión, el aprendizaje estratégico y una mayor responsabilidad de los estudiantes, al tiempo que promueve el uso crítico de los medios digitales (Anderson y Macleroy, 2016; Butler, 2016; Obeiah y Bataineh, 2016).

Por una parte, el e-PEL es un proyecto oficial que comenzó su implantación en 2009. Sin embargo, esta aplicación no se ha generalizado entre el profesorado y alumnado, ya que muy pocos centros emplean esta metodología. Por otra parte, el currículum vigente exige el desarrollo del plurilingüismo, que apenas se considera en el aula de idiomas. Dada la falta de estudios al respecto, es necesaria una investigación que analice la validez del e-PEL como instrumento de implementación del plurilingüismo con el fin de dinamizar este proyecto oficial. 
DESARROLLO DEL PLURILINGÜISMO A TRAVÉS DEL PORTFOLIO EUROPEO DE LAS LENGUAS ELECTRÓNICO (E-PEL) EN LA ESCUELA OFICIAL DE IDIOMAS: ESTUDIO DE UN CASO

\section{Metodología}

Este estudio cuantitativo analiza el desarrollo de los descriptores de plurilingüismo antes y después de la aplicación del e-PEL a la enseñanza-aprendizaje de idiomas en la EOI en cuatro grupos de alumnos de alemán de nivel A2.1, B1.1 y B1.2 (primer, tercer y cuarto curso) durante seis meses, de noviembre de 2015 a abril de 2016. El objetivo de esta investigación consiste en averiguar el grado de consecución de los descriptores de plurilingüismo del e-PEL, en lo que respecta a las actitudes y las actividades plurilingües de aprendizaje.

La recogida de datos se ha realizado a través de un cuestionario en español de 18 items, en el que las respuestas se han valorado mediante una escala Likert de dos puntos (Sí/Todavía no). Se ha utilizado el alfa de Cronbach para cuantificar el nivel de fiabilidad de la escala de medida para la magnitud inobservable (desarrollo del plurilingüismo mediante el e-PEL) construida a partir de las variables observadas (descriptores). El coeficiente rho de Spearman-Brown ha sido de 0,30 $(\mathrm{p}<0,05)$ y el coeficiente de fiabilidad asciende a 0,85 (0,81 en los ítems relativos a las actitudes y 0,89 en los referentes a las actividades), lo que confirma la fiabilidad del cuestionario.

\section{PARTICIPANTES}

La muestra de estudio está constituida por 109 alumnos, de los cuales el $32,11 \%$ son hombres y el $67,89 \%$ son mujeres, porcentajes similares a los alumnos de alemán (29,7\% de hombres y $69,3 \%$ de mujeres) y de los otros diez idiomas (39,4\% de hombres y 60,6\% de mujeres) impartidos en la EOI Alicante durante el curso 2015-2016. La diferencia estadística entre ambos sexos se puede deber a la mejor capacidad lingüística de las mujeres respecto a los hombres (OCDE, 2015). En lo que respecta a la edad, el 9,17\% tiene menos de 20 años, el 32,15\% se sitúa entre 20 y 30, el 35,76\% entre 30 y 40, el 18,34\% entre 40 y 50 y el 4,58\% más de 50. Según el test Kolmogorov-Smirnov (K-S), la hipótesis nula, H0, considera que la distribución de las variables seleccionadas proviene de una distribución normal. El nivel de significación obtenido en el test K-S ha ascendido a 0,20 para un nivel de significación de 0,05 , por lo que no rechazamos la hipótesis nula, ya que el p-valor es 0,20>0,05. Por tanto, según este test, podemos considerar que la distribución de los datos es normal, con concentración en torno a la media y dispersión en los extremos. A tal efecto, se ha llevado a cabo un muestreo incidental, seleccionando directa e intencionadamente los individuos de cuatro grupos de alumnos. Con el fin de evaluar la representatividad de la muestra se ha comparado la media muestral con la media poblacional de los alumnos de alemán y de los otros idiomas de la EOI en lo que respecta a sexo y edad con un nivel de confianza del 95\%. 


\section{CONTEXTO DE LA INVESTIGACIÓN}

Este estudio se ha llevado a cabo en la EOI Alicante. Esta institución educativa se define por su doble función, pedagógica y certificadora. El Ministerio de Educación, Cultura y Deporte establece en la Ley Orgánica 8/2013 para la mejora de la calidad educativa (LOMCE), en los artículos 59-62, los mínimos legales necesarios para la legislación de cada comunidad autónoma respecto a esta doble finalidad. En el artículo 59 se determina el objetivo de las enseñanzas de idiomas consistente en la capacitación de los alumnos para el uso adecuado de los idiomas en las etapas ordinarias del sistema educativo.

En el artículo 2 del Decreto 155/2007 del Consell, por el que se regulan las enseñanzas de idiomas de régimen especial en la Comunidad Valenciana, se destaca la dimensión pedagógica, ya que estas enseñanzas están destinadas a todos los que necesitan a lo largo de su vida adquirir o perfeccionar sus competencias en lenguas extranjeras y en valenciano. De igual manera, se hace hincapié en la vertiente certificadora, puesto que se posibilita la obtención de un certificado de su nivel de competencia. En particular, se pretende promover el estudio de las lenguas oficiales de los Estados miembros de la Unión Europea, del valenciano como lengua propia de la Comunidad Valenciana y del español para extranjeros, así como de otras lenguas que, por razones culturales, sociales o económicas sean de relevancia especial. La finalidad de las EOI se concreta en el desarrollo de la competencia comunicativa, asumiendo el enfoque de acción que propugna el MCERL. La aplicación del currículo tiene por objetivo fomentar la responsabilidad y la autonomía del alumno en el aprendizaje, la competencia plurilingüe y pluricultural y la dimensión intercultural, ya que estos aspectos repercuten en la mejora del aprendizaje.

\section{RESULTADOS Y ANÁlisis}

Los alumnos han cumplimentado un pretest y un postest con los descriptores de plurilingüismo del e-PEL con el fin de determinar su nivel de actitudes y actividades plurilingües antes y después de la experimentación. Los sujetos del estudio han respondido a los descriptores mediante dos respuestas: si y todavía no. La Tabla 1 muestra los datos obtenidos en el pretest y el postest: 
MARIO JESÚS MIRA-GIMÉNEZ

DESARROLLO DEL PLURILINGÜISMO A TRAVÉS DEL PORTFOLIO EUROPEO DE LAS LENGUAS ELECTRÓNICO (E-PEL) EN LA ESCUELA OFICIAL DE IDIOMAS: ESTUDIO DE UN CASO

TABLA 1

Consecución de los descriptores de plurilingüismo en el pretest y el postest y varianza

\begin{tabular}{|c|c|c|c|c|c|}
\hline \multirow[b]{2}{*}{ DESCRIPTORES DE PLURILINGÜISMO } & \multicolumn{2}{|c|}{ PREtest } & \multicolumn{2}{|c|}{ POSTEST } & \multirow[b]{2}{*}{ VARIANZA } \\
\hline & Sí & $\begin{array}{l}\text { TODAVÍA } \\
\text { NO }\end{array}$ & Sí & $\begin{array}{l}\text { TODAVÍA } \\
\text { NO }\end{array}$ & \\
\hline $\begin{array}{l}\text { 1. Estoy interesado por escuchar } \\
\text { mi lengua hablada por } \\
\text { personas con distinto acento. }\end{array}$ & $23,85 \%$ & $76,15 \%$ & $42,2 \%$ & $57,8 \%$ & $18,35 \%$ \\
\hline $\begin{array}{l}\text { 2. Estoy interesado en oír } \\
\text { otras lenguas, aunque } \\
\text { no las entienda. }\end{array}$ & $15,59 \%$ & $84,41 \%$ & $44,03 \%$ & $55,97 \%$ & $28,44 \%$ \\
\hline $\begin{array}{l}\text { 3. Si hiciera un viaje, estaría } \\
\text { interesado en poder saludar, } \\
\text { dar las gracias y utilizar } \\
\text { algunas expresiones similares } \\
\text { en la lengua del país. }\end{array}$ & $26,6 \%$ & $73,4 \%$ & $45,87 \%$ & $54,13 \%$ & $19,27 \%$ \\
\hline $\begin{array}{l}\text { 4. Merece la pena aprender } \\
\text { una lengua aunque sólo la } \\
\text { hablen pocas personas. }\end{array}$ & $16,51 \%$ & $83,49 \%$ & $48,62 \%$ & $51,38 \%$ & $32,11 \%$ \\
\hline $\begin{array}{l}\text { 5. El hecho de que en cada país } \\
\text { haya una lengua diferente } \\
\text { no es un problema para mí. }\end{array}$ & $22,93 \%$ & $77,07 \%$ & $45,87 \%$ & $54,13 \%$ & $22,94 \%$ \\
\hline $\begin{array}{l}\text { 6. Hablar la lengua de } \\
\text { otra gente me permite } \\
\text { comprender mejor su } \\
\text { cultura (literatura, cine, } \\
\text { tradiciones, etc.). }\end{array}$ & $28,44 \%$ & $71,56 \%$ & $73,4 \%$ & $26,6 \%$ & $44,96 \%$ \\
\hline $\begin{array}{l}\text { 7. Estoy interesado por } \\
\text { aprender una nueva lengua } \\
\text { aunque sea sólo para } \\
\text { poderla entender o leer. }\end{array}$ & $30,27 \%$ & $69,73 \%$ & $83,49 \%$ & $16,51 \%$ & $53,22 \%$ \\
\hline $\begin{array}{l}\text { 8. Conocer más de una lengua } \\
\text { me ayuda a aprender otras. }\end{array}$ & $26,6 \%$ & $73,4 \%$ & $89 \%$ & $11 \%$ & $62,4 \%$ \\
\hline $\begin{array}{l}\text { 9. Aprender una nueva } \\
\text { lengua me permite ver el } \\
\text { mundo de otra manera. }\end{array}$ & $31,19 \%$ & $68,81 \%$ & $57,8 \%$ & $42,2 \%$ & $26,61 \%$ \\
\hline $\begin{array}{l}\text { 10. He traducido textos de } \\
\text { una lengua a otra. }\end{array}$ & $25,68 \%$ & $74,32 \%$ & $89 \%$ & $11 \%$ & $63,32 \%$ \\
\hline 11. He actuado como intérprete. & $19,26 \%$ & $80,74 \%$ & $87,15 \%$ & $12,85 \%$ & $67,89 \%$ \\
\hline
\end{tabular}


DESARROLLO DEL PLURILINGÜISMO A TRAVÉS DEL PORTFOLIO EUROPEO DE LAS LENGUAS ELECTRÓNICO (E-PEL) EN LA ESCUELA OFICIAL DE IDIOMAS: ESTUDIO DE UN CASO

\begin{tabular}{|c|c|c|c|c|c|}
\hline \multirow[b]{2}{*}{ DESCRIPTORES DE PLURILINGÜISMO } & \multicolumn{2}{|c|}{ PRETEST } & \multicolumn{2}{|c|}{ Postest } & \multirow[b]{2}{*}{ VARIANZA } \\
\hline & Sí & $\begin{array}{c}\text { ToDAvía } \\
\text { NO }\end{array}$ & Sí & $\begin{array}{c}\text { ToDAvía } \\
\text { NO }\end{array}$ & \\
\hline $\begin{array}{l}\text { 12. He resumido un texto } \\
\text { original en una lengua } \\
\text { a partir de un texto } \\
\text { en otra lengua. }\end{array}$ & $12,84 \%$ & $87,16 \%$ & $84,41 \%$ & $15,59 \%$ & $71,57 \%$ \\
\hline $\begin{array}{l}\text { 13. He trabajado con archivos } \\
\text { que contenían textos en } \\
\text { diferentes lenguas. }\end{array}$ & $24,77 \%$ & $75,23 \%$ & $84,41 \%$ & $15,59 \%$ & $59,64 \%$ \\
\hline $\begin{array}{l}\text { 14. He dado charlas/conferencias } \\
\text { en las que he cambiado } \\
\text { de lengua/en las que he } \\
\text { resumido en otra lengua. }\end{array}$ & $11 \%$ & $89 \%$ & $83,49 \%$ & $16,51 \%$ & $72,49 \%$ \\
\hline $\begin{array}{l}\text { 15. He participado en } \\
\text { conversaciones en diferentes } \\
\text { lenguas y con personas que } \\
\text { se entendian mutuamente. }\end{array}$ & $11,92 \%$ & $88,08 \%$ & $88,08 \%$ & $11,92 \%$ & $76,16 \%$ \\
\hline $\begin{array}{l}\text { 16. He dado charlas/ } \\
\text { conferencias en una lengua } \\
\text { usando notas escritas en } \\
\text { una lengua diferente. }\end{array}$ & $6,42 \%$ & $93,58 \%$ & $84,41 \%$ & $15,59 \%$ & $77,99 \%$ \\
\hline $\begin{array}{l}\text { 17. He escuchado programas } \\
\text { de televisión en los que } \\
\text { podía cambiar de lengua } \\
\text { procurando no perder el hilo. }\end{array}$ & $7,33 \%$ & $92,67 \%$ & $84,41 \%$ & $15,59 \%$ & $77,08 \%$ \\
\hline $\begin{array}{l}\text { 18. He contado a mi profesor } \\
\text { de una lengua lo que he } \\
\text { hecho o hago cuando } \\
\text { aprendo otra lengua. }\end{array}$ & $10,09 \%$ & $89,91 \%$ & $89 \%$ & $11 \%$ & $78,91 \%$ \\
\hline
\end{tabular}

En el pretest, satura en todas las variables Todavía no, con porcentajes altos (entre 68,81\% y 93,58\%). En el cuestionario se ha seguido la misma sucesión de descriptores que figura en el e-PEL. Si estudiamos su carácter, podemos dividirlos en dos ámbitos. Los ítems 1-9 hacen referencia a las actitudes respecto al plurilingüismo, mientras que los descriptores 10-18 se centran en actividades plurilingües de aprendizaje, de tal manera que constituyen dos dimensiones diferentes de la competencia plurilingüe. Si nos atenemos a su diferente naturaleza, se aprecian divergencias en la dispersión de los porcentajes, según se observa en la Figura 1: 
DESARROLLO DEL PLURILINGÜISMO A TRAVÉS DEL PORTFOLIO EUROPEO DE LAS LENGUAS ELECTRÓNICO (E-PEL) EN LA ESCUELA OFICIAL DE IDIOMAS: ESTUDIO DE UN CASO

FIGURA 1

Dispersión de datos en el Pretest

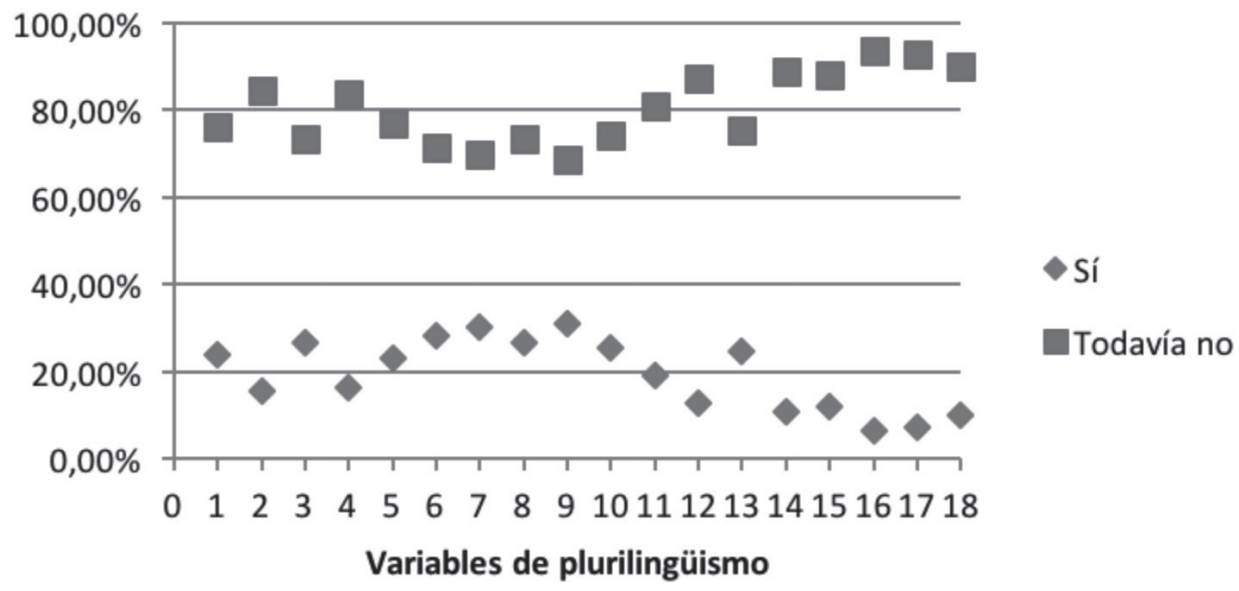

En el pretest, en los descriptores 1-9 de actitud respecto al plurilingüismo, se observan valores que oscilan entre el $84,41 \%$ y el $68,81 \%$ en la variable Todavía no, mientras que en los ítems 10-18 los porcentajes son mayores, entre el 93,58\% y el $74,32 \%$. Este hecho se observa también en la media de alumnos que responden Todavía no en el pretest: en los descriptores referidos a las actitudes, el promedio asciende al 75,33\%, mientras que en los ítems de actividades plurilingües de aprendizaje la media se sitúa en el 85,63\%, lo que supone más de un 10\% de diferencia. En el postest se produce un cambio de tendencia. En los cinco primeros descriptores, la mayoría de alumnos sigue contestando Todavía no, si bien los valores son inferiores a los registrados en el pretest, oscilando entre el 57,8\% y el 51,38\%. El aspecto que ha registrado el peor resultado con una cantidad mayor de alumnos que todavía no lo han conseguido es el descriptor que se refiere al interés por escuchar su lengua hablada por personas con distinto acento. Con un porcentaje similar, los otros cuatro descriptores con mayor número de discentes que todavía no han conseguido ese objetivo también hacen referencia a actitudes lingüísticas relativas a otros idiomas que no forman parte del ámbito personal o de estudio. Es relevante el descriptor 5 que se refiere al hecho del plurilingüismo dentro de un país. El estudio se ha llevado a cabo en Alicante y en la Comunidad Valenciana conviven dos lenguas oficiales: español y valenciano. La mayoría de alumnos tienen el español como lengua materna. Su situación de lengua mayoritaria condiciona la sensibilidad por las lenguas minoritarias y minorizadas.

Sin embargo, en los ítems 6-9, más de la mitad de los encuestados contesta Sí, con resultados entre el $89 \%$ y el 57,8\%. Esta respuesta satura en todos los descriptores de actividades plurilingües, con porcentajes que se sitúan entre el $89 \%$ y el 83,49\%, según se observa en la Figura 2: 
FiguRA 2

Dispersión de datos en el Postest

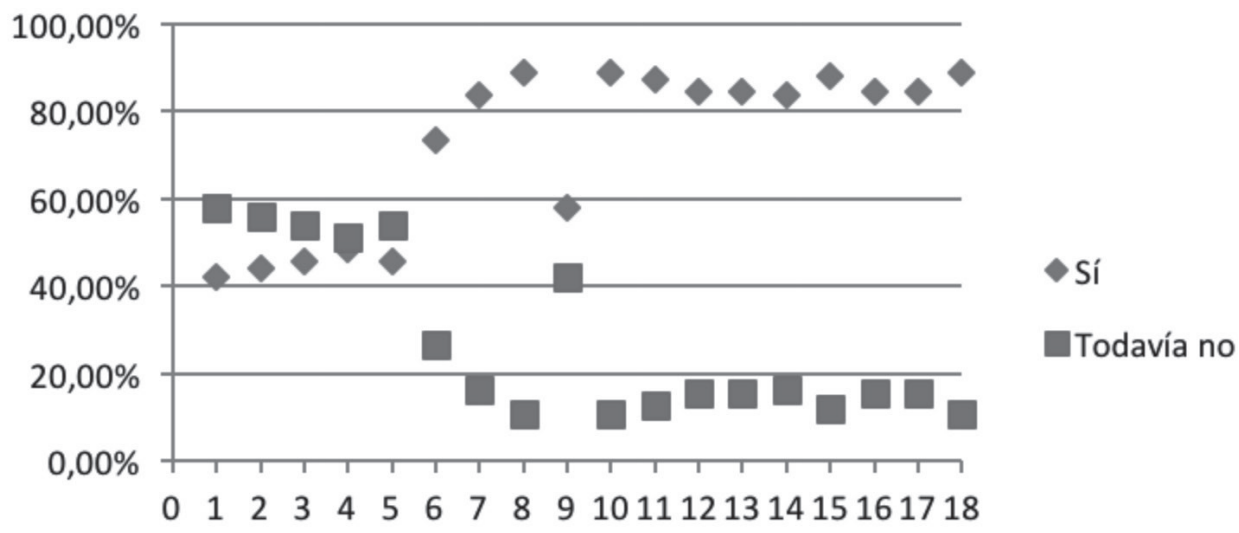

Variables de plurilingüismo

Esta tendencia se confirma también en el promedio de alumnos que responden $S i ́$ en el postest: en los descriptores referidos a las actitudes, la media se sitúa en el 58,92\%, mientras que en los descriptores de actividades plurilingües de aprendizaje la media se sitúa en el 86,04\%, lo que implica una diferencia del $27,12 \%$, tal y como se aprecia en la Figura 3:

FIGURA 3

Consecución de los descriptores de plurilingüismo

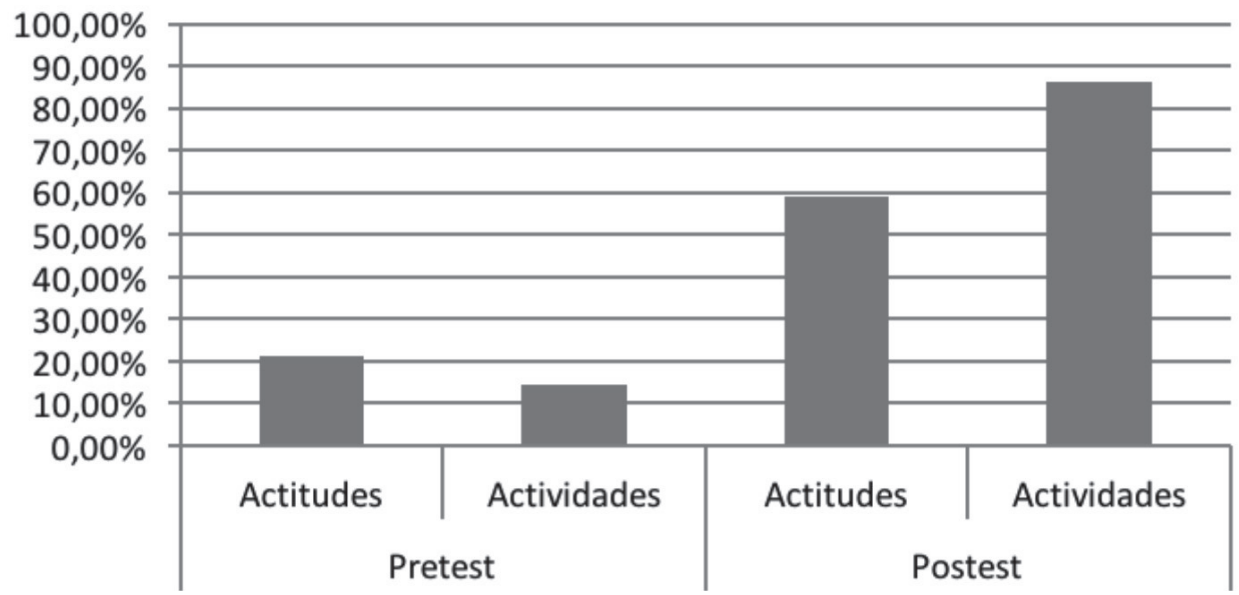


Desagregados los datos del pretest por sexo, satura la respuesta Todavía no entre los hombres con un porcentaje del 89,24\% en actitudes y el $81,67 \%$ en actividades, mientras que se invierten los porcentajes en el caso de las mujeres, con el 72,36\% y el $87,33 \%$, respectivamente. Si desagregamos los porcentajes por edad, no hay grandes variaciones en el promedio de alumnos que responden Todavía no en el pretest: el 83,57\% en los menores de 20 años, el 76,29\% entre 20 y 30 años, el $77,81 \%$ entre 30 y 40 , el $75,13 \%$ entre 40 y 50 y el 80,86\% entre los mayores de $50(\mathrm{C}=0,121$, Sig. $=0,277)$. El análisis factorial exploratorio concede al Test KMO el valor 0,80, el valor 621,56 a la prueba de esfericidad de Bartlett y 0,000 al nivel de significación. Por consiguiente, se confirma que el modelo es adecuado.

Si desagregamos los resultados del postest por sexo, en lo que respecta a las actitudes, las mujeres registran una media del 67,78\% en actitudes y el 90,07\% en actividades, por el $46,05 \%$ y el $74,19 \%$ entre los hombres, respectivamente. Desagregados los datos por edad, observamos mayores variaciones en la respuesta Si en los descriptores referidos a las actitudes en los diferentes grupos de edad: el 42,3\% en los menores de 20 años, el 57,2\% entre 20 y 30 años, el 63,4\% entre 30 y 40, el $68,7 \%$ entre 40 y 50 y el 49,4\% entre los mayores de 50 (Coeficiente de correlación $=0,157$, Sig. $=0,321)$. El análisis factorial exploratorio concede al Test KMO el valor 0,70, el valor 589,51 a la prueba de esfericidad de Bartlett y 0,000 al nivel de significación. Por tanto, se confirma la adecuación del modelo.

Si analizamos la varianza de resultados entre el pretest y el postest, observamos que en todos los ítems se registra un aumento de alumnos que consiguen alcanzar el objetivo del descriptor, si bien el crecimiento es dispar en los dos ámbitos de los ítems: en los descriptores de actitudes el aumento se sitúa entre el $62,4 \%$ y el 18,35\%, mientras que, en actividades plurilingües, entre el 78,91\% y el 63,32\%. Conviene reseñar que el desarrollo siempre es menor en los ítems del primer ámbito que los del segundo, excepto en los descriptores 8 y 10, según se aprecia en la Figura 4: 

EN LA ESCUELA OFICIAL DE IDIOMAS: ESTUDIO DE UN CASO

FIGURA 4

Dispersión entre el pretest y el postest

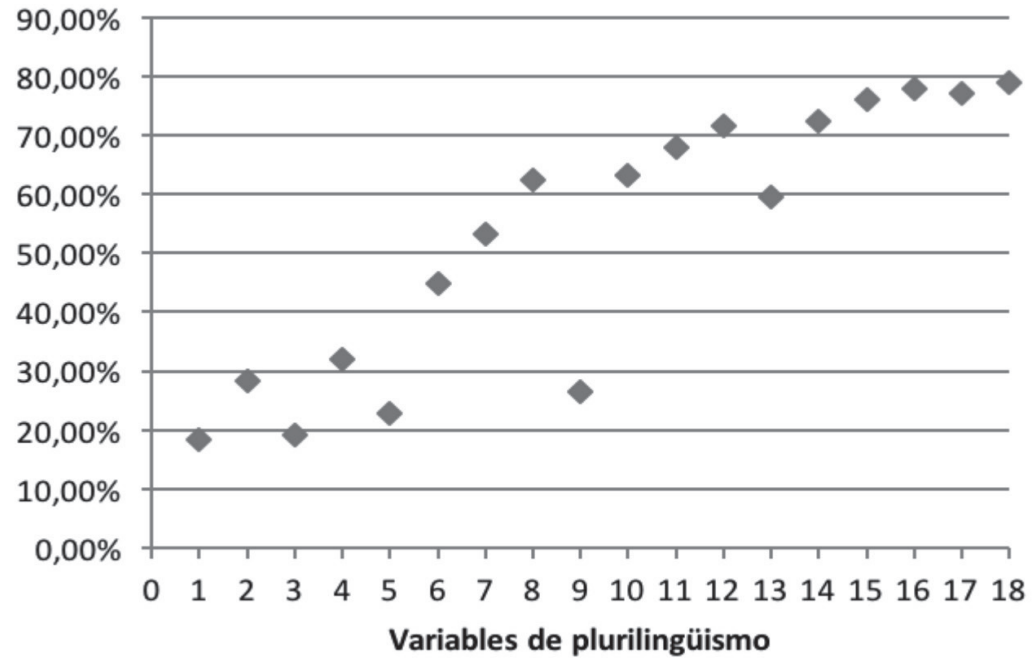

Varianza

\section{Discusión}

A tenor de los resultados, se puede concluir que el e-PEL fomenta el desarrollo del plurilingüismo a través del cambio de actitudes y la utilización de actividades plurilingües de aprendizaje. Sin embargo, es necesario destacar su mayor efectividad en el segundo ámbito durante los seis meses de investigación. A pesar del desarrollo en lo que respecta a actitudes, sería necesario más tiempo para implementar actividades que fomenten el interés por otras culturas y lenguas, que desde el punto de vista pragmático, ya sea social o económico, tienen mayor relevancia. Del análisis de los datos se desprende que las mujeres y los grupos de edad cercanos a la media han experimentado una mejoría más significativa en la competencia plurilingüe. Este aspecto podría estar motivado por la mejor competencia lingüística de las mujeres (OCDE, 2015), lo cual podría implicar más interés por otras lenguas y culturas, y la mayor necesidad de la competencia plurilingüe y pluricultural en el ámbito profesional actual (Yanaprasart, 2016).

La aportación del e-PEL al desarrollo del plurilingüismo se demuestra por el cambio de tendencia registrado en los descriptores 6-8 y 10-18 con un aumento superior al 30\%. Estos descriptores hacen referencia a las actividades plurilingües llevadas a cabo con el e-PEL. Sin embargo, los alumnos siguen sin conseguir los descriptores 1-5, los cuales se centran en actitudes. En el descriptor 9, que se refiere al cambio de percepción del mundo, la mayoría de discentes confirman la consecución del objetivo, si bien el porcentaje de los que no lo hacen es cercano al 50\%. Por consiguiente, el desarrollo de actitudes positivas hacia el plurilingüismo necesita más tiempo de 
DESARROLLO DEL PLURILINGÜISMO A TRAVÉS DEL PORTFOLIO EUROPEO DE LAS LENGUAS ELECTRÓNICO (E-PEL) EN LA ESCUELA OFICIAL DE IDIOMAS: ESTUDIO DE UN CASO

implementación y de medidas complementarias para su desarrollo como intercambios lingüísticos o actividades con las personas extranjeras residentes.

Respecto a los datos por edad, la varianza superior al $57 \%$ registrada en los grupos entre 20 y 50 años demuestra que los alumnos de estos intervalos de edad se encuentran más sensibilizados ante la necesidad del plurilingüismo en la sociedad actual. En el grupo de menores de 20 años, sería conveniente introducir el trabajo con el e-PEL en la enseñanza primaria y secundaria a fin de fomentar la conciencia plurilingüe en este sector de población. En el grupo de más de 50 años, los resultados pueden explicarse por la falta de situaciones interculturales y plurilingües en la España de la enseñanza reglada y la sociedad de este segmento de la población. El trabajo para revertir este fenómeno debería hacer hincapié en la globalización y la necesidad de actitudes tolerantes hacia otras culturas y lenguas.

En conclusión, el e-PEL constituye la herramienta para poner en práctica el cambio metodológico que propugna el MCERL en la transmisión de la competencia plurilingüe, especialmente en el ámbito de las actividades. Sin embargo, son necesarias medidas complementarias para el desarrollo de actitudes, así como más tiempo de aplicación, no solo en el contexto estudiado, sino también en la enseñanza obligatoria.

\section{REFERENCIAS BIBLIOGRÁFICAS}

Álvarez, A. (2016). El análisis contrastivo cultural como método de partida en el aula con estudiantes sinohablantes. Recuperado de http://cvc.cervantes.es/Ensenanza/biblioteca_ele/asele/pdf/25/25_0117.pdf.

Anderson, J. y Macleroy, V. (2016). Reflections and implications for multilingual digital literacy. En J. Anderson y V. Macleroy (Eds.). Multilingual Digital Storytelling: Engaging Creatively and Critically with Literacy (pp. 261-267). Oxon/Nueva York: Routledge Research in Education.

Angelova, M. y Zhao, Y. (2016). Using an online collaborative project between American and Chinese students to develop ESL teaching skills, cross-cultural awareness and language skills. Computer Assisted Language Learning, 29 (1), 167-185. DOI: $10.1080 / 09588221.2014 .907320$.

Bârliba, D. M. (2015). Remarks about the relationship between language and intercultural education. The International Conference 'Education and Creativity for a Knowledge-Based Society' - Social, Political \& Humanistic Sciences - IXth Edition. Bucarest, 19-21 de noviembre.

Bien, O.; Merten, S. y Schnotz, W. (2015). The Individual Theories of Teachers about Vocabulary Work with Mono-and Plurilingual Students in the German Language Classroom. En W. Schnotz, A. Kauertz, H. Ludwig, A. Müller y J. Pretsch (Eds.). Multidisciplinary Research on Teaching and Learning (pp. 129-145). Basingstoke: Palgrave Macmillan UK.

Block, D. (2016). The impact of globalisation, internationalisation and migration on the use and vitality of Catalan in secondary school and higher education settings. Language, Culture and Curriculum, 29 (1), 107-116. DOI: 10.1080/07908318.2016.1132661.

Butler, Y. G. (2016). Self-assessment of and for young learners' foreign language learning. En M. Nikolov (Ed.). Assessing Young Learners of English: Global and Local Perspectives (pp. 291-315). Springer International Publishing. 
Byram, M. (1992). Culture et éducation en langue étrangère. París: Hatier.

Collins, L. y Muñoz, C. (2016). The Foreign Language Classroom: Current Perspectives and Future Considerations. The Modern Language Journal, 100 (S1), 133-147. DOI: 10.1111/ modl.12305.

Cummins, J. (1983). Interdependencia lingüística y desarrollo educativo de los niños bilingües. Infancia y aprendizaje, 6 (21), 37-68. DOI: 10.1080/02103702.1983.10821962.

Daryai-Hansen, P.; Gerber, B.; Lörincz, I.; Haller, M.; Ivanova, O.; Krumm, H. J. y Reich, H. H. (2015). Pluralistic approaches to languages in the curriculum: the case of French-speaking Switzerland, Spain and Austria. International Journal of Multilingualism, 12 (1), 109-127. DOI: 10.1080/14790718.2014.948877.

Decreto 155/2007, de 21 de septiembre, por el que se regulan las enseñanzas de idiomas de régimen especial en la Comunitat Valenciana y se establece el currículo del nivel básico y del nivel intermedio. DOCV, n. $^{\circ} 5605$, de 24 de septiembre de 2007.

Dezuanni, M. y Monroy-Hernández, A. (2012). "Prosumidores interculturales»: creación de medios digitales globales entre jóvenes [Prosuming” across Cultures: Youth Creating and Discussing Digital Media across Borders]. Comunicar, 38 (XIX), 59-66. DOI: http:// dx.doi.org/10.3916/C38-2012-02-06.

Díaz, A. R. (2016). Developing Interculturally-Oriented Teaching Resources in CFL: Meeting the Challenge. En R. Moloney y H. L. Xu. Exploring Innovative Pedagogy in the Teaching and Learning of Chinese as a Foreign Language (pp. 115-135). Singapur: Springer Singapore.

Dumbavescu, D. G. y Merino, S. (2015). Del saber estar con uno mismo al saber estar con el otro: el componente afectivo como parte esencial de la formación docente. En Y. Morimoto, M. V. Pavón Lucero y R. Santamaría Martínez. La enseñanza de ELE centrada en el alumno (pp. 323-329). Madrid: ASELE.

Fortanet-Gómez, I. (2013). CLIL in Higher Education. Towards a Multilingual Language Policy. Bristol: Multilingual Matters.

Garbarino, S. (2015). Intercomprensione, informatica, interazione, internazionalizzazione, interdisciplinarità: Miriadi di «i» per la formazione al plurilinguismo. En D. Spiță, M. Lupu, D. Nica y I. Nica (Eds.). Les approches plurielles dans l'éducation aux langues: l'intercompréhension, en présence et en ligne (pp. 81-94). Iași: Editura Universității Alexandru Ioan Cuza.

García, J. A. y Moreno, I. (Coords.) (2014). Escuela, diversidad cultural e inclusión. Madrid: La Catarata.

González, M. (2015). La enseñanza de las lenguas extranjeras en España y Holanda: un estudio comparado (Tesis inédita de doctorado). Universidad Complutense, Madrid.

Hancock, A. (2014). Language education policy in multilingual Scotland: opportunities, imbalances and debates. Language Problems and Language Planning, 38 (2), 167-191. DOI: 10.1075/lplp.38.2.04han.

Hanesová, D. (2015). Plurilingual and intercultural Awareness of Future teachers. New Education Review, 42 (4), 79-90. DOI: 10.15804/TNER.2015.42.4.06.

Iñesta, E. M. y Pascual, J. (2015). Didáctica para el plurilingüismo en la formación de maestros: Estudio empírico desde el prácticum. Aula Abierta, 43 (2), 94-101. DOI: 10.1016/j. aula.2014.12.001.

Jessner, U.; Allgäuer-Hackl, E. y Hofer, B. (2016). Emerging Multilingual Awareness in Educational Contexts: From Theory to Practice. Canadian Modern Language Review, 1-26. DOI: http://dx.doi.org/10.3138/cmlr.274600. 
MARIO JESÚS MIRA-GIMÉNEZ

DESARROLLO DEL PLURILINGÜISMO A TRAVÉS DEL PORTFOLIO EUROPEO DE LAS LENGUAS ELECTRÓNICO (E-PEL) EN LA ESCUELA OFICIAL DE IDIOMAS: ESTUDIO DE UN CASO

Kabilan, M. K. (2016). Using Facebook as an e-portfolio in enhancing pre-service teachers' professional development. Australasian Journal of Educational Technology, 32 (1), 19-31. DOI: http://dx.doi.org/10.14742/ajet.2052.

Komorowska, H. (2016). The European Language Policy and the teaching profession in the $21^{\text {st }}$ century. Studia Linguistica Universitatis Iagellonicae Cracoviensis, 2015 (3), 135150. DOI: $10.4467 / 20834624$ SL.15.014.3935.

Konstantinidou, D. T. y Pampouri, A. A. (2015). European policies on lifelong learning and the use of EQF, Europass and Counseling in Higher Education Institutions in Greece. En Proceedings of the 15th International Academic Conference (pp. 589-598). International Institute of Social and Economic Sciences (IISES). DOI: 10.20472/IAC.2015.015.100.

Lancaster, N. K. (2016). Stakeholder Perspectives on CLIL in a Monolingual Context. English Language Teaching, 9 (2), 148-177. DOI: http://dx.doi.org/10.5539/elt.v9n2p148.

Ley Orgánica 8/2013 para la mejora de la calidad educativa (LOMCE). BOE, n. ${ }^{\circ}$ 295, de 10 de diciembre de 2013.

Lindorfer, B. (2016). Inner Language Spaces: Migration and Plurilingualism from a Psycholinguistic Perspective. En B. Mersmann y H. G. Kippenberg (Eds.). The Humanities between Global Integration and Cultural Diversity (pp. 211-226). Berlin/Boston: De Gruyter/ Fritz Thyssen Stiftung für Wissenschaftsförderung.

Little, D. y Erickson, G. (2015). Learner Identity, Learner Agency, and the Assessment of Language Proficiency: Some Reflections prompted by the Common European Framework of Reference for Languages. Annual Review of Applied Linguistics, 35, 120-139. DOI: $10.1017 /$ S0267190514000300.

López, M. y Abello, C. (2016). El pluralismo y la diversidad en los contenidos (inter)culturales de los libros de texto para la enseñanza de inglés en los centros bilingües de Andalucía. Círculo de Lingüística Aplicada a la Comunicación, 65, 199-222. DOI: 10.5209/ rev_CLAC.2016.v65.51986.

López-Rocha, S. (2016). Intercultural Communicative Competence: Creating Awareness and Promoting Skills in the Language Classroom. En C. Goria, O. Speicher y S. Stollhans (Eds.). Innovative language teaching and learning at university: enhancing participation and collaboration (pp. 105-112). Dublín: Research-publishing.net.

Madrid, D. (2014). Integrating the European portfolio in a competency-based teacher education approach. Utrecht Studies in Language and Communication, 27 (2014), 35-58. Recuperado de http://0-literature.proquest.com.fama.us.es/searchFulltext.do?i$\mathrm{d}=\mathrm{R} 05007423 \&$ divLevel=0\&area=abell\&forward=critref_ft.

Manzano, B. (2015). Clil in three Spanish monolingual communities: the examples of Extremadura, Madrid and La Rioja. ELIA, 15, 135-158. DOI: http://dx.doi.org/10.12795/ elia.2015.i15.07.

Méndez, M. d. C. (2016). Intercultural reflection through the Autobiography of Intercultural Encounters: students' accounts of their images of alterity. Language and Intercultural Communication, 1-28. DOI: 10.1080/14708477.2016.1159693.

Messina, C. (2015). Evaluar la conciencia intercultural: una autoevaluación guiada. MarcoELE: Revista de Didáctica Español Lengua Extranjera, 20 (2015), 1-3. Recuperado de http:// marcoele.com/descargas/20/messina-conciencia_intercultural.pdf.

Meyer, M.; Prediger, S.; César, M. y Norén, E. (2016). Making Use of Multiple (Non-shared) First Languages: State of and Need for Research and Development in the European Language Context. En R. Barwell, P. Clarkson, A. Halai, M. Kazima, J. Moschkovich, 
N. Planas, ... y M. V. Ubillús (Eds.). Mathematics Education and Language Diversity (pp. 47-66). Springer International Publishing.

Mira-Giménez, M. J. (2015). Implementación del Portfolio Europeo de las Lenguas Electrónico: estudio de un caso. RISTI-Revista Ibérica de Sistemas e Tecnologias de Informação, 16, 46-59. DOI: http://dx.doi.org/10.17013/risti.16.46-59.

Mira-Giménez, M. J. (2016). Un nou enfocament en l'aprenentatge d'idiomes amb les TiC: l'e-PEL. Revista d'Innovació Docent Universitària, 8, 1-14.

Newby, D. (2007). European Portfolio for Student Teachers of Languages: A reflection tool for language teacher education. Estrasburgo: Consejo de Europa.

Newby, D.; Fenner, A. B. y Jones, B. (Eds.) (2011). Using the European portfolio for student teachers of languages. Graz: Consejo de Europa.

Nicolás, S. y Abril, S. (2015). Autonomous Learning and Self-Assessment through the European Language Portfolio (ELP): A Pilot Study on Primary Education. The Journal of Language Teaching and Learning, 5 (2), 37-53. Recuperado de http://journal.jltl.org/ index.php/jltl/article/view/386/96.

Nieuwboer, C. y van't Rood, R. (2016). Learning language that matters: A pedagogical method to support migrant mothers without formal education experience in their social integration in Western countries. International Journal of Intercultural Relations, 51, 29-40. DOI: 10.1016/j.ijintrel.2016.01.002.

North B. (2016). Developing CEFR illustrative descriptors of aspects of mediation. International Online Journal of Education and Teaching (IOJET), 3 (2), 132-140. Recuperado de http://www.iojet.org/index.php/IOJET/article/view/125/131.

Obeiah, S. F. y Bataineh, R. F. (2016). The Effect of Portfolio-Based Assessment on Jordanian EFL Learners' Writing Performance. Bellaterra Journal of Teaching \& Learning Language E Literature, 9 (1), 32-46. DOI: http://dx.doi.org/10.5565/rev/jtl3.629.

Pérez, M. L. (2016). Are teachers ready for CLIL? Evidence from a European study. European Journal of Teacher Education, 39 (2), 202-221. DOI: 10.1080/02619768.2016.1138104.

Real Decreto 126/2014, de 28 de febrero, por el que se establece el currículo básico de la Educación Primaria. $B O E$, n. $^{\circ}$ 52, de 1 de marzo de 2014.

Schärer, R. (2015). Revisiting the principles and functions of the European Language Portfolio with a focus on tertiary education. ВЕСТНИКМОСКОВСКОГОГОСУ ААРСТВЕННОГО АИНГВИСТИЧЕСКОГО УНИВЕРСИТЕТА, 14 (725), 28-47. Recuperado de http:// cyberleninka.ru/article/n/revisiting-the-principles-and-functions-of-the-european-language-portfolio-with-a-focus-on-tertiary-education.

Serrador, R. (2015). Intercambios y competencia intercultural: nuevos enfoques y contextos en la didáctica de lengua extranjera. En N. Ibarra-Rius, J. Ballester-Roca, M. L. Carrió-Pastor y F. Romero-Forteza (Eds.). Retos en la adquisición de las literaturas y de las lenguas en la era digital (pp. 553-559). Valencia: Editorial Universidad Politécnica de Valencia.

Taylor, C. (1993). El multiculturalismo y la política de reconocimiento. México DF: Fondo de Cultura Económica.

Yanaprasart, P. (2016). Managing Language Diversity in the Workplace: Between 'One Language Fits All' and 'Multilingual Model in Action'. Universal Journal of Management, 4 (3), 91-107. DOI: 10.13189/ujm.2016.040302.

Zorba, M. G. y Arıkan, A. (2016). A Study Of Anatolian High Schools' 9th Grade English Language Curriculum In Relation To The CEFR. Uşak Üniversitesi Eğitim Araştırmalar Dergisi, 2 (2), 13-24. Recuperado de http://dergipark.ulakbim.gov.tr/usakead/article/ download/5000182837/5000163672. 